\title{
Problemas Socioemocionales en Niños con Discapacidad Auditiva, Discapacidad Visual y Desarrollo Típico
}

\section{Socioemotional Problems in Children with Hearing Impairment, Visual Impairment and Typical Development}

\author{
Catalina Santa Cruz* \\ Victoria Espinoza \\ Elisa Hohlberg \\ Pontificia Universidad Católica de Chile, Chile
}

\begin{abstract}
Los niños con discapacidad presentan mayor riesgo de presentar problemas socioemocionales. En el presente estudio se comparó la presencia de problemas emocionales en preescolares con discapacidad visual, discapacidad auditiva y desarrollo típico en cuanto a los problemas totales, externalizantes e internalizantes, así como respecto de síndromes específicos. El grupo con discapacidad sensorial presenta significativamente más problemas emocionales en la escala total y la escala de problemas externalizantes, que el grupo de desarrollo típico. Distinguiendo por tipo de discapacidad presentada, el análisis arrojó que los tres grupos difieren significativamente solo en las escalas total y externalización. Finalmente, se analizó la prevalencia de rangos de riesgo y clínicos para los tres grupos principales. Aun cuando las diferencias no son estadísticamente significativas, optan por ser mayores en los grupos con discapacidad sensorial. Los resultados se discuten en torno a la importancia de la detección y prevención de problemas socioemocionales tempranamente, lo cual es fundamental para promover una mejor calidad de vida de las personas con discapacidad, además de evitar enfermedades mentales en el futuro.
\end{abstract}

Descriptores: Discapacidad auditiva; Discapacidad visual; Problemas socioemocionales; Problemas internalizantes; Problemas externalizantes.

Children with disabilities are at greater risk of presenting social-emotional problems. In the present study, the presence of emotional problems in preschool children with visual impairment, hearing impairment, and typical development was compared in total, externalizing and internalizing problems and respect to specific syndromes. The group with sensory disabilities presents significantly more emotional problems on the total scale and externalizing problems than the typical developmental group. Distinguishing by type of disability, the analysis showed that the three groups differ significantly only in the total and externalization scales. Finally, we analyzed the prevalence of risk and clinical ranges for the three main groups. Even when the differences are not statistically significant, they choose to be greater in the groups with sensory disabilities. The results are discussed around the importance of early detection and prevention of socio-emotional problems, which is essential to promote a better quality of life for people with disabilities and avoid mental illnesses in the future.

Keywords: Hearing impairment; Visual impairment; Socio-emotional problems; Externalizing problems; Internalizing problems. 


\section{Introducción}

Se ha estimado que aproximadamente un $20 \%$ de los niños, niñas y adolescentes sufren algún tipo de enfermedad mental (World Health Organization, 2020). Diversos estudios han reportado que entre un $5 \%$ y un $26 \%$ de la población presenta problemas emocionales y de comportamiento. La presencia de este tipo de problemas en niños y adolescentes puede generar importantes dificultades respecto de su funcionamiento social, académico y emocional (Brauner y Stephens, 2006). Limitando sus oportunidades de integración social en la adolescencia, afectando el desarrollo de sus relaciones con pares, padres, profesores e incluso con sus parejas (Naciones Unidas, 2014).

Por otra parte, un adecuado desarrollo de las habilidades emocionales ha demostrado tener un alto impacto sobre la vida de las personas, a nivel de las relaciones interpersonales, el bienestar psicológico, el desempeño académico, la conducta y el bienestar general (Eisenberg, 2006; Fernández-Berrocal y Ruiz Aranda, 2008; Lopes, 2005). Además, se ha observado que las personas con un mayor desarrollo de estas habilidades describen una menor presencia de sintomatología depresiva (Goldenberg, Matheson y Mantler, 2006).

La salud mental, entre otras cosas, considera el estado emocional y respuestas conductuales en niños, niñas y adolescentes (NNA). De esta forma, los problemas emocionales y conductuales representan una serie de dificultades asociadas a la presencia de problemas de carácter internalizante y externalizante. Los problemas internalizantes hacen referencia a dificultades de orden personal, dentro de las que encontramos trastornos como la depresión, la ansiedad, el retraimiento y las quejas somáticas. Al contrario, los problemas externalizantes son aquellos que involucran conflictos con otras personas y afectan sus expectativas respecto de los niños. En este grupo se puede encontrar los trastornos de comportamiento agresivo y desafiante (Achenbach y Rescorla, 2001). Por lo tanto, se considera que la salud mental, se asocia a la ausencia o niveles limitados de problemas emocionales y conductuales.

Por otra parte, se ha demostrado que la presencia de problemas internalizantes y externalizantes en la infancia, puede predecir la presencia de futuros problemas de salud mental en la adolescencia (Jones, 2013; Mesman et al., 2001). Por lo que es fundamental indagar respecto de su presencia en etapas escolares tempranas. En este marco, la presente investigación tiene como objetivo general describir las características asociadas a la salud mental, entendida desde los problemas emocionales, de alumnos que presentan discapacidad y desarrollo típico (DT).

\section{Revisión de la literatura}

\subsection{Problemas emocionales y discapacidad}

Diversos estudios han propuesto la existencia de una mayor prevalencia de problemas emocionales y conductuales en niños con discapacidad (Alimovic, 2013), lo que podría suponer mayor riesgo de sufrir las consecuencias sociales, académicas y emocionales descritas para la población general. En el caso de niños con discapacidad sensorial, se ha encontrado mayor presencia de problemas sociales y habilidades de reconocimiento y comprensión de las emociones (Dyck et al., 2004; Maes y Grietens, 2004).

A continuación, se presentan evidencias relativas a la presencia de problemas emocionales en niños y adolescentes con discapacidad auditiva (DA) y discapacidad visual (DV). 
Niños y niñas con discapacidad auditiva. Se ha observado que niños y adolescentes con DA presentan mayores problemas emocionales y conductuales que sus pares oyentes (Dammeyer, 2018; Stevenson et al.,, 2015; Vissers y Hermans, 2018). Se ha descrito que la prevalencia de problemas de salud mental en niños sordos o hipoacúsicos es entre dos a cuatro veces mayor que en el caso de niños de DT (Dammeyer, 2009; van Eldik et al., 2004; van Gent et al., 2007).

En un estudio desarrollado en Alemania con 238 niños y adolescentes sordos de entre 4 a 18 años, se pudo observar que los niños sordos presentan mayor presencia de problemas emocionales y conductuales, tanto de carácter internalizante como externalizante. Un $41 \%$ de los niños puntuaron dentro del rango clínico, existiendo mayores problemas de ansiedad y depresión entre los adolescentes de 12 a 18 años que entre los niños de 4 a 11 años (van Eldik et al., 2004).

En un metaanálisis desarrollado por Stevenson y otros (2015) se analizaron los hallazgos de treinta y tres estudios que evaluaron los comportamientos, emociones y las relaciones interpersonales de niños y adolescentes sordos e hipoacúsicos. Ellos encontraron que los niños con DA puntuaban entre un cuarto y un tercio de desviación estándar más que los niños oyentes, indicando mayor presencia de problemas. Por otra parte, se describió que el área con mayores dificultades era aquella relativa a "problemas con pares". Los resultados de los distintos estudios analizados indicaron que, tanto en función de la percepción de sus padres como respecto de sus propias valoraciones, los estudiantes con DA se perciben más aislados socialmente, con mayor tendencia a la agresividad y con una menor autoestima respecto de sus pares oyentes. Los estudios analizados incluyeron niños y adolescentes desde los 18 meses hasta los 21 años. Se realizaron análisis para determinar si el tamaño del efecto cambiaba según la edad de los participantes, y en la mayoría de los casos se pudo ver que declinaba a medida que se avanzaba en edad. Sin embargo, los autores sugieren la necesidad de establecer estudios longitudinales para determinar esta relación.

Más específicamente, van Eldik y otros (2004) propusieron la existencia de diferencias significativas entre niños y adolescentes con DA y sus pares oyentes respecto de la escalas total de problemas, la escala de problemas externalizantes y de problemas internalizantes. Ellos también describieron la presencia de diferencias significativas respecto de síndromes específicos, como lo son el retraimiento, la ansiedad y depresión, los problemas sociales, los problemas de pensamiento, los problemas atencionales, la conducta delictiva y el comportamiento agresivo. El único síndrome donde no se observaron diferencias significativas fue el relativo a quejas somáticas.

Por otra parte, también existen estudios que plantean que no existen diferencias en el desarrollo de problemas emocionales entre niños con DA y niños oyentes. En una investigación desarrollada por Khan y otros (2005) se analizó la existencia de diferencias en el desarrollo de problemas emocionales en niños con DA con y sin implantes cocleares y niños oyentes de entre dos a siete años de edad. Los resultados evidenciaron que no existían diferencias significativas entre ninguno de los grupos. De acuerdo con esta investigación, Huber y Kipman (2011) compararon la salud mental de adolescentes con DA usuarios de implante coclear y adolescentes oyentes. Los investigadores no encontraron diferencias significativas entre los grupos, sin embargo, estos resultados podrían no ser representativos para el caso de niños sordos sin implante coclear. 
Basándose en múltiples estudios, Dammeyer (2018) describe una serie de factores de riesgo que podrían explicar esta mayor tendencia de los niños con DA a presentar problemas psicosociales y de salud mental, siendo los más importantes el desarrollo de las habilidades lingüísticas, el tipo de educación recibida, el apoyo parental y el uso de implantes cocleares. Respecto del desarrollo de las habilidades lingüísticas, se propone que, en muchos casos la falta o pérdida de audición se traduce en un menor desarrollo del lenguaje, lo que aumenta la presencia de problemas emocionales. En este sentido, se deduce que aquellos niños que presentan un adecuado desarrollo de las habilidades lingüísticas sean éstas en lengua de señas o a través del lenguaje oral, presentan una menor tendencia a desarrollar de problemas de salud mental. Por otra parte, el efecto del tipo de escuela a la que asisten los niños está determinado por el tipo de comunicación que presentan. Los niños que tienen un adecuado desarrollo de la lengua de señas presentan menor prevalencia de problemas emocionales cuando estudian en escuelas especiales, en cambio los niños que tienen un mayor desarrollo del lenguaje oral presentan menores problemas cuando se encuentran integrados en escuelas regulares. En definitiva, la habilidad de los niños para comunicarse con sus pares es fundamental para prevenir problemas de salud mental. El apoyo de los padres, por su parte, se describe como el factor menos estudiado, pero también relacionado con la comunicación. Barker y su equipo (2009), concluyen que la asociación entre lenguaje y problemas conductuales depende del nivel de lenguaje desarrollado, especialmente durante la primera infancia. Su estudio muestra cómo aquellos niños con discapacidad auditiva presentan más problemas de tipo conductual y mayores dificultades con el lenguaje oral, las que a su vez se asocian a un menor tiempo de interacciones entre padres e hijos(as). Se propone que los niños con DA provenientes de familias que reportan tener buenos niveles de comunicación temprana presentan una mejor calidad de vida. Al respecto, Dammeyer (2018) plantea que el uso de implantes cocleares y ayudas técnicas también tiene un impacto a través del lenguaje. En este sentido, el grado en que el implante coclear favorece las habilidades lingüísticas de los niños es lo que determina su influencia en la menor presencia de problemas de salud mental. Por su parte, las ayudas técnicas parecen no favorecer suficientemente el desarrollo del lenguaje, existiendo una asociación entre éstas y un menor nivel de bienestar. De todas formas, el autor plantea que muchos de estos factores deben ser más estudiados para contar con conclusiones generalizables.

\subsection{Niños y niñas con discapacidad visual}

En el caso de los niños y adolescentes con DV, también se ha observado una mayor prevalencia de problemas emocionales y de comportamiento respecto de niños de DT (Alimovic, 2013; Dale y Salt, 2008). Investigaciones previas han reportado una prevalencia de entre un $20 \%$ a un $49 \%$ respecto de la presencia de problemas emocionales y conductuales en niños con DV (Ophir-Cohen et al., 2005; Tirosh et al., 1998).

Un estudio donde se comparó la presencia de problemas emocionales en niños de 4 a 11 años con DV y niños de DT, se pudo observar que éstos últimos presentaban una cantidad significativamente menor de problemas emocionales y de comportamiento. Existiendo diferencias significativas tanto respecto de los problemas internalizantes como externalizantes. Respecto de la presencia de trastornos específicos, solo se encontraron diferencias significativas respecto de las quejas somáticas, los problemas sociales y de atención. Estas diferencias indicaban mayor presencia de problemas en el caso de los niños con DV (Alimovic, 2013). 
Por otra parte, en una investigación desarrollada con 111 niños con DV pertenecientes a escuelas especiales (Mohamed et al., 2019), se observó una alta presencia de problemas respecto de las competencias sociales. Alrededor de un tercio de los participantes manifestó un rango límite o clínico respecto de los problemas de comportamiento, reportándose en un $27,7 \%$ de los niños evaluados rangos límites o clínicos respecto de la presencia de problemas internalizantes, y un 32,7\% en rangos límites o clínicos en relación con la presencia de problemas externalizantes.

Uno de los principales factores de riesgo que podrían explicar una presencia mayor de problemas emocionales y conductuales en niños con DV, es un desarrollo descendido de las habilidades sociales. En un estudio donde se analizaron las interacciones sociales de niños de entre 9 a 12 meses, se pudo observar que en comparación con niños sin DV, los niños ciegos exhibían un repertorio más limitado de expresiones faciales, eran menos receptivos, tenían menos intentos de iniciar la contacto con sus madres o de presentar quejas o requerimientos hacia ellas (Tröster y Brambring, 1992). También se ha señalado que los niños ciegos tienen un menor desarrollo de la Teoría de la Mente, además se ha observado que presentan un acceso limitado a las relaciones causales existentes entre las expresiones faciales de las personas y los contextos que las inducen (Roch-Levecq, 2006). Santa-Cruz y Hohlberg (2019) estudiaron la autorregulación de alumnos preescolares con DV a través de la evaluación de las Funciones Ejecutivas. Las autoras concluyen que hay una tendencia a que los niños con DV, presenten menor Memoria de Trabajo y Funciones Ejecutivas en general. También se ha podido determinar la existencia de una dificultad específica respecto del reconocimiento de emociones, no así respecto de la comprensión de las mismas (Dyck et al., 2004). Por otra parte, Runji'c y otros (2015) estudiaron a 39 adolescentes con DV de 13 a 17 años y concluyeron que aquellos estudiantes que presentaban mayor desarrollo de sus habilidades sociales manifestaron menor presencia de problemas de comportamiento. Los niños con menores problemas sociales presentaban una mayor autoestima, se sentían menos ansiosos y tenían menos problemas con los adultos. En este sentido Wright y Torrey (2000) encontraron una correlación consistente entre escalas prosociales y problemas de comportamiento al evaluar a un grupo de niños con DV de quinto y sexto grado.

Sin embargo, también existe evidencia relativa a la inexistencia de diferencias en el desarrollo emocional y el comportamiento de niños ciegos y niños de DT. En un estudio que tuvo por objetivo comparar la prevalencia de problemas emocionales en niños ciegos y niños de DT de entre 11 y 14 años, se pudo observar que los niños ciegos presentaban menor puntuación que el grupo de comparación respecto de los problemas emocionales, específicamente respecto de la ansiedad, depresión, aislamiento y problemas de atención; y no presentaban diferencias significativas con el grupo control respecto del comportamiento agresivo, problemas de pensamiento, quejas somáticas, problemas sociales y problemas internalizantes y externalizantes (Demir et al., 2014).

En un estudio realizado con adolescentes con DV se pudo observar que, si bien los estudiantes ciegos no diferían significativamente del grupo control respecto de la frecuencia de depresión, síntomas de estrés y problemas en sus relaciones con sus padres y hermanos, los adolescentes con DV reportaban tener menos amigos y encuentros con personas de su edad, así como mayor sensación de soledad y dificultades para hacer amigos. Las niñas con DV presentaban una menor autoestima, un peor desempeño escolar y habilidades sociales más descendidas (Huurre y Aro, 1998). 
Por último, es importante considerar el grado de DV que presentan los estudiantes. En el meta-análisis publicado por Pinquart y Pfeiffer (2011), se establece una relación negativa entre el grado de discapacidad y el grado de bienestar psicológico de los individuos. Esto implica que, a mayor grado de discapacidad, menor es el grado de bienestar psicológico reportado.

En definitiva, si bien la investigación internacional presenta evidencias relativas a una mayor presencia de problemas emocionales y del comportamiento en niños con discapacidad sensorial, también existen evidencias que indican lo contrario. El presente estudio busca indagar respecto de los distintos aspectos del desarrollo emocional y del comportamiento de niños y niñas con DA y DV en la etapa preescolar, en comparación con el desarrollo de niños sin discapacidad sensorial.

Para responder al objetivo principal se examinó las diferencias en problemáticas socioemocionales de alumnos que están en proceso de adquisición de la lectura. Las preguntas que orientan el análisis son las siguientes: (1) ¿Presentan los alumnos con discapacidad sensorial mayores problemas socioemocionales, en las escalas total, de internalización y externalización y en los síndromes específicos, que los alumnos de DT en edad preescolar?, (2) ¿Hay diferencias entre los alumnos con DA, DV y DT respecto al tipo y magnitud de problemas que presentan? y (3) ¿Hay diferencias en cuanto al tipo de comunicación utilizada en el caso de los alumnos con DA y del grado de discapacidad en el caso de los alumnos con DV que expliquen diferencias en la cantidad de problemas reportados? (4) ¿Cuál es la prevalencia de los rangos clínicos respecto a los tres grupos comparados?

Las hipótesis, basadas en la literatura revisada, son: (1) se aprecian mayores problemas socioemocionales en general, expresado en la escala total del instrumento de evaluación y específicos, expresados en las escalas que evalúan problemas internalizantes y externalizantes, así como síndromes particulares, en el grupo que presenta discapacidad sensorial, en comparación con sus pares de DT.

La primera hipótesis específica es que el grupo que presenta DV presenta una mayor proporción de problemáticas de tipo internalizante, en comparación con el grupo que presenta DA y DT. La segunda hipótesis específica corresponde a que el grupo con DA presenta una mayor proporción de problemáticas de tipo externalizante, en comparación con el grupo que presenta DV y DT. Por último, se hipotetiza que hay diferencias de acuerdo a las características particulares de cada grupo de alumnos con discapacidad sensorial, esto implica que, la tercera hipótesis indica que habrá mayor problemática en las escalas para el grupo que se comunica mediante lengua de señas versus comunicación oral en el caso de los niños y niñas con DA. Y la cuarta hipótesis señala que el grupo con DV reportará mayores problemas en aquellos alumnos que presentan discapacidad total, versus el grupo que presenta discapacidad parcial.

\section{Método}

Para responder las preguntas de investigación se utilizó el paradigma cuantitativo, con un diseño de estudio no experimental transversal de tipo correlacional. 


\section{Sujetos}

La muestra contó con un total de 192 estudiantes, de los cuales un 47,91\% se identifica con el sexo masculino. La edad media muestral fue de 71,38 (DE $=11,178)$ meses. La muestra considera, alumnos que presentan discapacidad visual o auditiva, ya sea en grado parcial o total, además de un grupo de alumnos que presentan DT, el cual considera alumnos que no presentan necesidades educativas especiales, de acuerdo a lo reportado por los apoderados. Esta muestra cuenta con la particularidad de haber sido intencionada para poder evaluar trayectorias del aprendizaje de matemáticas y lenguaje en alumnos con discapacidad sensorial, por lo cual uno de los criterios de inclusión fue estar en proceso de adquisición de la lectura. Lo anterior implica que, en muchos casos, no corresponde con un grado escolar regular o edad promedio similar en el caso de los alumnos con discapacidad. Asimismo, un criterio de exclusión, para toda la muestra, fue el tener comorbilidad diagnosticada al tiempo del estudio. En el Cuadro 1, se pueden observar las características demográficas de la muestra.

\section{Cuadro 1}

Estadísticos descriptivos de la muestra

\begin{tabular}{|c|c|c|c|c|c|c|c|c|}
\hline & \multicolumn{2}{|c|}{ DT } & \multicolumn{2}{|c|}{ DV } & \multicolumn{2}{|c|}{ DA } & \multicolumn{2}{|c|}{ Total } \\
\hline & $\mathbf{M}$ & DE & $\mathbf{M}$ & $\mathrm{DE}$ & $\mathbf{M}$ & DE & $\mathbf{M}$ & DE \\
\hline $\begin{array}{l}\text { Edad } \\
\text { (meses) }\end{array}$ & 68,333 & 4,631 & 76,346 & 15,694 & 91,235 & 19,661 & 71,383 & 11,178 \\
\hline & $\mathbf{N}$ & $\%$ & n & $\%$ & $\mathbf{n}$ & $\%$ & $\mathbf{N}$ & $\%$ \\
\hline Sexo & & & & & & & & \\
\hline Hombre & 66 & 34,375 & 14 & 7,292 & 12 & 6,250 & 92 & 47,917 \\
\hline Mujer & 83 & 43,229 & 12 & 6,25 & 5 & 2,604 & 100 & 52,083 \\
\hline GD & & & & & & & & \\
\hline Parcial & & 10 & 38,462 & & & & 10 & 38,462 \\
\hline Total & & 16 & 61,538 & & & & 16 & 61,538 \\
\hline $\mathrm{TC}$ & & & & & & & & \\
\hline Oral & & & & & 5 & 29,412 & 5 & 29,4112 \\
\hline $\mathrm{LS}$ & & & & & 12 & 70,588 & 12 & 70,588 \\
\hline
\end{tabular}

Nota. DT = Desarrollo típico, DV=Discapacidad visual, DA= Discapacidad auditiva, M= Media, $\mathrm{DE}=$ Desviación estándar, $\mathrm{n}=$ Número de participantes del grupo, $\mathrm{N}=$ Número de participantes de la muestra total, $\mathrm{GD}=$ Grado de discapacidad, $\mathrm{TC}=$ Tipo de comunicación.

\section{Instrumentos}

$\mathrm{El}$ instrumento utilizado para la recogida de datos fue el Child-Behavior Checklist 6-18 (CBCL) desarrollado por Achenbach y Rescorla (2001), corresponde a un formulario que permite evaluar competencias, funcionamiento adaptativo y problemas que experimentan niños, niñas y adolescentes durante su etapa escolar, de acuerdo a lo informado por sus padres, apoderados o cuidadores que conozcan al estudiante en un ambiente familiar. Este instrumento ha sido utilizado en diversas investigaciones tanto nacionales (Lecannelier et al., 2014; Leiva Bahamondes y Andrade, 2018; Reynolds et al., 2018; Vicente et al., 2012) como internacionales (Ivanova et al., 2007; Rocha et al., 2013), y ha sido utilizada en grupos de niños, niñas y jóvenes con y sin discapacidad (Alimovic, 2013; Demir et al., 2014a; Dovgan et al., 2019; Ivanova et al., 2007; Khan et al., 2005; Lecannelier et al., 2014; Leiva Bahamondes y Andrade, 2018; Maes y Grietens, 2004). La completación del instrumento demora entre 15 y 20 minutos. Los autores sugieren que el instrumento puede ser utilizado desde los cinco años si los niños van a ser evaluados nuevamente a los seis, lo cual corresponde al caso de esta investigación. 
De acuerdo con la asesoría recibida por parte de los editores de CBCL (Achenbach System of Empirically Based Assessment (ASEBA)), comunicación personal, 13 de febrero, 2018) se utilizó la versión adaptada para Chile de la sección correspondiente a problemas socioemocionales y conductuales. El instrumento cuenta con 113 ítems que describen características del alumno o alumna. El adulto que responde debe considerar si esa característica es "muy cierta o a menudo cierta” (2 puntos), de "alguna manera o algunas veces cierta” (1 punto) o, “no es cierta” (o puntos). La calificación debe basarse en lo observado durante los últimos seis meses.

Los resultados están organizados en torno a ocho síndromes, los cuales son obtenidos a través de las normas de publicación del instrumento. De acuerdo con sus autores, los síndromes refieren a un conjunto de problemas que tienden a ser concurrentes. Los síndromes fueron nombrados en pos de resumir los síntomas y problemas que agrupan. Estos a su vez, pueden ser agrupados en tres escalas generales, relativas conductas externalizantes, problemas internalizantes y una escala general o total (Achenbach y Rescorla, 2001).

En el Cuadro 2 se observan los ocho síndromes y se exponen tres ítems por cada uno para ilustrar el tipo de conducta o problema que comprende. Por último, se menciona el índice de confiabilidad de cada una de las escalas, de acuerdo a lo que se indica en el manual del instrumento.

\section{Cuadro 2}

Descripción y confiabilidad de las escalas

\begin{tabular}{|c|c|c|}
\hline SÍNDROME & ÍTEMS DE EJEMPLO & $\begin{array}{c}\alpha \text { DE } \\
\text { CRONBACH }\end{array}$ \\
\hline Ansioso-Depresivo & $\begin{array}{l}\text { (a) Teme ir al colegio } \\
\text { (b) Llora constantemente } \\
\text { (c) Se siente demasiado culpable }\end{array}$ & 0,84 \\
\hline Retraído-Depresivo & $\begin{array}{l}\text { (a) No habla } \\
\text { (b) Prefiere estar solo } \\
\text { (c) Disfruta poco }\end{array}$ & 0,80 \\
\hline Quejas Somáticas & $\begin{array}{l}\text { (a) Tiene pesadillas } \\
\text { (b) Se siente mareado } \\
\text { (c) Tiene dolores de cabeza }\end{array}$ & 0,78 \\
\hline Problemas Sociales & $\begin{array}{l}\text { (a) No habla } \\
\text { (b) Prefiere estar solo } \\
\text { (c) Disfruta poco }\end{array}$ & 0,82 \\
\hline Problemas de Pensamiento & $\begin{array}{l}\text { (a) Repite acciones } \\
\text { (b) No puede dejar de pensar ciertas cosas } \\
\text { (c) Se hace daño }\end{array}$ & 0,78 \\
\hline Problemas Atencionales & $\begin{array}{l}\text { (a) Actúa como si fuese menor } \\
\text { (b) Le cuesta terminar tareas } \\
\text { (c) Es impulsivo(a) }\end{array}$ & 0,86 \\
\hline Transgresión de Reglas & $\begin{array}{l}\text { (a) Miente o hace trampa } \\
\text { (b) Dice garabatos o malas palabras } \\
\text { (c) Roba }\end{array}$ & 0,85 \\
\hline Conducta Agresiva & $\begin{array}{l}\text { (a) Destruye sus cosas } \\
\text { (b) Es desobediente } \\
\text { (c) Ataca a otras personas }\end{array}$ & 0,94 \\
\hline
\end{tabular}

Nota. Recuperado de Asenbach y Rescorla (2001, p. 101).

A continuación, se describen las tres escalas generales que reporta el instrumento y sus respectivos índices de confiabilidad: 
- Problemas Totales: Esta escala comprende los ocho síndromes antes descritos en el Cuadro 2, por lo que su puntaje bruto puede oscilar entre los o y 240 puntos, siendo que a mayor puntaje mayor índices de problemas socioemocionales en general. Presenta una confiabilidad de $\alpha=0,94$.

- Problemas Internalizantes: Esta escala agrupa los síndromes AnsiosoDepresivo, Retraído-Depresivo y Quejas Somáticas. Debe su nombre a que las conductas y problemas descritos se relacionan con el sujeto mismo. Su confiabilidad es de $\alpha=0,91$.

- Problemas Externalizantes: Esta escala considera los síndromes Transgresión de Reglas y Conducta Agresiva. Su nombre indica que los problemas y conductas son en relación con otras personas y a lo que se espera en el comportamiento de un niño o niña. El $\alpha$ de Cronbach es igual a 0,94.

Cada uno de los síndromes y escalas generales pueden ser transformados a un puntaje $\mathrm{T}$ correspondiente al puntaje de la norma estandarizada publicada por ASEBA, la cual distingue por grupo etáreo y sexo. Chile no cuenta con una norma nacional, por lo que, siguiendo las recomendaciones de la editorial, se utilizó la correspondiente a la sociedad 2, la cual incluye la norma estándar del instrumento, y es adecuada para países como Croacia, Grecia e Italia. En el caso de los síndromes, se considera que un puntaje T de entre 65 y 69, el niño o niña está en el rango límite, esto implica que sus síntomas no son clínicos, pero hay una tendencia a ello. Por consiguiente, si el puntaje es mayor a 69, se considera que el síndrome alcanzó una intensidad clínica. En el caso de las escalas generales, se considera que el rango clínico está entre los puntajes $\mathrm{T}=60-63$ y el rango clínico cuando se superan los 63 puntos.

Es necesario acotar que los autores del instrumento no recomiendan utilizar los puntajes $\mathrm{T}$ de los síndromes para análisis estadísticos continuos, sino que solo en términos de prevalencia. Los autores señalan que para realizar comparaciones es necesario transformar los puntajes brutos obtenidos por todos los participantes a puntajes estándar. En esta investigación se transformaron los puntajes obtenidos por toda la muestra a puntaje Z, para evitar confusiones entre los puntajes $\mathrm{T}$ propios de la norma del instrumento. Por lo tanto, los puntajes $\mathrm{T}$ se utilizaron para las escalas generales y para obtener las prevalencias de las categorías límite y clínicas. Y, por su parte, los puntajes Z se utilizaron para hacer comparaciones entre los grupos experimentales para las escalas relativas de síndromes específicos.

\section{Procedimiento}

El presente estudio utilizó datos correspondientes a la primera ola de evaluación del estudio longitudinal "Considerando la Diversidad en Poblaciones Diversas: de las Políticas a las Intervenciones en Educación Especial", llevado a cabo por el Centro de Justicia Educacional de la Pontificia Universidad Católica de Chile (PUC) y financiado por ANID. El proyecto fue aprobado por el Comité de Ética de la PUC y fue aceptado por los directivos de las escuelas contactadas, además de contar con el consentimiento informado de apoderados y asentimiento informado de los estudiantes que participaron del estudio. Los alumnos fueron evaluados entre los meses de abril y mayo del año 2018, siendo en este caso los apoderados quienes respondieron el cuestionario socioemocional, de acuerdo con la percepción que tenían respecto de sus hijos. 


\section{Plan de Análisis}

En este estudio se comparan los niveles de problemas socioemocionales reportados por apoderados de alumnos agrupados en torno a tres características principales a saber; DA, DV y DT.

El análisis considera reportar diferencias en los problemas socioemocionales reportados por la escala, desde lo general a lo particular. Por lo tanto, se estructura considerando primero si la población que presenta discapacidad sensorial difiere de aquella de DT en cuanto a la Escala Total, Escala de Internalización y Escala de Externalización del CBCL. Luego, se comparan estos dos grupos respecto a dimensiones específicas, dadas por las escalas de síndromes que reporta el instrumento. Todos estos análisis fueron realizados mediante la prueba T de Student.

Posteriormente, el análisis considera si hay diferencias entre los tres grupos principales, es decir DA, DV y DT, en las mismas escalas que se mencionan anteriormente. Cada una de las comparaciones se realizó mediante pruebas de ANOVA unidireccionales.

Para terminar con esta línea de análisis, se realizaron comparaciones específicas para los grupos con discapacidad sensorial. Esto implica que los alumnos con DA fueron analizados en cuanto al tipo de comunicación que utilizan, es decir si utilizan comunicación oral o lengua de señas. Por su parte, los alumnos con DV fueron analizados en cuanto al grado de discapacidad que presentan, es decir si reportan una discapacidad parcial o total. En el caso de las comparaciones intragrupo con discapacidad, estas se efectuaron mediante pruebas T de Student.

Considerando que los tamaños muestrales no son equivalentes y en algunas de las comparaciones no superan los cinco alumnos, es que además de los estadísticos, se reporta el tamaño del efecto asociado a cada prueba. Esto permite profundizar en los resultados obtenidos, aun cuando estos provengan de grupos pequeños.

Por último, se utilizó la categorización de las distintas escalas de CBCL para reportar el índice de prevalencia de los rangos clínicos y de riesgo, para los tres grupos y se comparó la proporción de estos criterios diagnósticos en cuanto a su significancia estadística mediante la prueba de Chi-cuadrado.

\section{Resultados}

La evaluación de los supuestos necesarios para realizar el análisis estadístico antes mencionado indica que de los grupos de comparación solo el que presenta DA tiene un outlier (a más de dos DE del promedio grupal) en la escala de internalización, evaluado mediante inspección visual del boxplot. Dada la robustez de los estadísticos utilizados, se realizaron los análisis considerando el outlier. De acuerdo a la inspección visual realizada por medio de gráficos $Q-Q$, todos los grupos presentan una distribución normal de los datos. Por último, se aprecia normalidad en la homogeineidad de la varianza de los grupos de comparación principal, de acuerdo al test de Levene de homogeneidad de varianzas $(\mathrm{p}=0,08)$.

\subsection{Discapacidad sensorial Vs. desarrollo típico}

$\mathrm{Al}$ comparar los promedios de los alumnos que presentan discapacidad sensorial, con los que no presentan este tipo de discapacidad, se observa diferencias estadísticamente 
significativas, en la cual los alumnos sin discapacidad tienen en promedio 5,311 IC 95\% [$8,842,-1,778]$ puntos menos de la escala de problemas total $(\mathrm{t}(194)=-3,436, \mathrm{p}=0,001$, $\mathrm{d}=0.548)$.

Como se aprecia en el Cuadro 3, tanto en las escalas de internalización como externalización, el grupo que no reporta discapacidad presenta consistentemente menores puntajes respecto del grupo que reporta discapacidad sensorial, diferencias que son estadísticamente significativas

\section{Cuadro 3}

Comparación grupo discapacidad sensorial versus desarrollo típico

\begin{tabular}{|c|c|c|c|c|c|c|c|c|c|}
\hline & \multirow{2}{*}{ GRUPO } & \multirow{2}{*}{$\mathbf{N}$} & \multirow{2}{*}{ Media } & \multirow{2}{*}{ DE } & \multirow{2}{*}{ EE } & \multicolumn{2}{|c|}{ ICC } & \multirow{2}{*}{$\mathrm{T}(\mathrm{P})$} & \multirow{2}{*}{ D } \\
\hline & & & & & & LI & $\mathbf{L S}$ & & \\
\hline \multirow{2}{*}{ Escala Total } & $\overline{\mathrm{DT}}$ & 153 & 52,294 & 10,871 & 0,879 & \multirow{2}{*}{$-8,842$} & \multirow{2}{*}{$-1,779$} & $-3,436$ & \multirow{2}{*}{0,549} \\
\hline & DS & 43 & 57,605 & 8,336 & 1,271 & & & $-0,001$ & \\
\hline \multirow{2}{*}{$\begin{array}{l}\text { Escala } \\
\text { Internalización }\end{array}$} & DT & 153 & 51,412 & 10,635 & 0,86 & \multirow{2}{*}{$-7,225$} & \multirow{2}{*}{$-0,138$} & $-2,049$ & \multirow{2}{*}{0,364} \\
\hline & DS & 43 & 55,093 & 9,546 & 1,456 & & & $-0,042$ & \\
\hline \multirow{2}{*}{$\begin{array}{l}\text { Escala } \\
\text { Externalización }\end{array}$} & DT & 153 & 53,85 & 9,725 & 0,786 & \multirow{2}{*}{$-6,657$} & \multirow{2}{*}{$-0,155$} & $-2,066$ & \multirow{2}{*}{0,366} \\
\hline & DS & 43 & 57,256 & 8,891 & 1,356 & & & $-0,04$ & \\
\hline
\end{tabular}

Nota. DT $=$ Desarrollo típico, $\mathrm{DS}=$ Discapacidad sensorial, $\mathrm{N}=$ Número de participantes de la muestra total, $\mathrm{DE}=$ Desviación estándar, Error Estándar de la media, $\mathrm{ICC}=95 \%$ del Intervalo de confianza de la diferencia, LI=Límite Inferior, LS=Límite Superior

En cuanto a las diferencias obtenidas en cada uno de los síndromes, se obtiene que en todas las comparaciones el grupo de DT obtiene menores puntajes y por ende menores problemas, como se puede observar en la Figura 1. Sin embargo, estas diferencias son estadísticamente significativas solo para los síndromes Quejas Somáticas $(\mathrm{t}(194)=-2.217$, $\mathrm{p}=0,028, \mathrm{~d}=-0,143)$, Problemas Sociales $(\mathrm{t}(194)=-4.025, \mathrm{p}<0,001, \mathrm{~d}=-0.383)$, Problemas de Pensamiento $(\mathrm{t}(194)=-2.064, \mathrm{p}=0.044, \mathrm{~d}=-0.695)$, Problemas Atencionales $(\mathrm{t}(194)=-$ $3.356, \mathrm{p}=0.001, \mathrm{~d}=-0.412)$.

\section{Figura 1}

Escalas de Sindromes CBCL, según grupos discapacidad sensorial y desarrollo típico

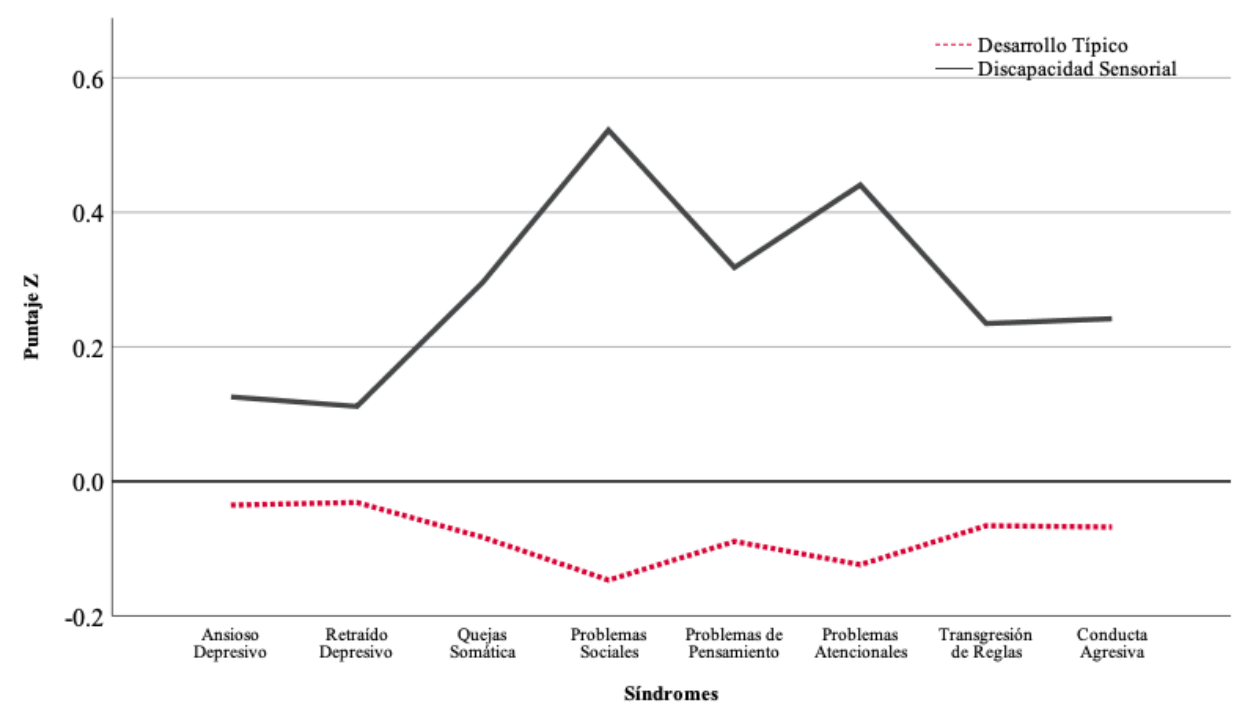




\subsection{Discapacidad auditiva, discapacidad visual y desarrollo típico}

Al diferenciar el grupo que presenta discapacidad sensorial, considerando si ésta es de tipo auditivo o visual, el análisis de varianza indica, como se observa en el Cuadro 4, que hay diferencias estadísticamente significativas tanto en la escala total, como en la de externalización, no así en la de internalización. Al hacer comparaciones post-hoc utilizando el método Tukey, solo las diferencias de medias en los grupos de DT y DA son estadísticamente significativas ( $\operatorname{diff}=6,327, \mathrm{p}=0,027$ ) para la escala de externalización, tendencia que se repite para la escala total en la cual nuevamente solo los grupos que consideran alumnos con DT y DA presentan diferencias significativas (diff $=7,059$, $\mathrm{p}=0,023)$.

\section{Cuadro 4}

Comparación grupo discapacidad visual, discapacidad auditiva y desarrollo típico

\begin{tabular}{|c|c|c|c|c|c|c|c|c|c|}
\hline & & & & & & IC & $\bar{M}$ & & \\
\hline \multirow{4}{*}{ Escala Total } & GRUPO & $\mathbf{N}$ & MEDIA & DE & EE & LI & $\mathbf{L S}$ & $\mathbf{F}(\mathbf{P})$ & $\eta^{2}$ \\
\hline & DT & 153 & 52,29 & 10,871 & 0,879 & 50,56 & 54,03 & 4,792 & \\
\hline & DV & 26 & 56,46 & 7,834 & 1,536 & 53,3 & 59,63 & $-0,009$ & 0,047 \\
\hline & DA & 17 & 59,35 & 9,007 & 2,184 & 54,72 & 63,98 & & \\
\hline \multirow{3}{*}{$\begin{array}{l}\text { Escala } \\
\text { Externalización }\end{array}$} & DT & 153 & 53,85 & 9,725 & 0,786 & 52,3 & 55,4 & 3,479 & \\
\hline & DV & 26 & 55,35 & 7,155 & 1,403 & 52,46 & 58,24 & $-0,033$ & 0,035 \\
\hline & DA & 17 & 60,18 & 10,608 & 2,573 & 54,72 & 65,63 & & \\
\hline \multirow{3}{*}{$\begin{array}{l}\text { Escala } \\
\text { Internalización }\end{array}$} & DT & 153 & 51,41 & 10,635 & 0,86 & 49,71 & 53,11 & 2,264 & \\
\hline & DV & 26 & 55,85 & 10,593 & 2,077 & 51,57 & 60,12 & $-0,107$ & 0,033 \\
\hline & DA & 17 & 53,94 & 7,846 & 1,903 & 49,91 & 57,98 & & \\
\hline
\end{tabular}

Nota. DT = Desarrollo típico, DV = Discapacidad visual, DA=Discapacidad Auditiva, N= Número de participantes de la muestra total, $\mathrm{DE}=$ Desviación estándar, Error Estándar de la media, ICC= 95\% del Intervalo de confianza de la diferencia, LI=Límite Inferior, LS=Límite Superior.

En la Figura 2 se aprecia que los alumnos que reportaron no tener discapacidad sensorial presentan consistentemente menos problemas en todas las escalas que evalúan síndromes. Por su parte, los alumnos con DV superan a los otros dos grupos en las escalas que evalúan problemas internalizantes, mientras que los alumnos con DA superan en su índice de problemas en todas las escalas restantes a los otros dos grupos de comparación. El ómnibus test muestra que hay diferencias significativas entre los grupos en las prueba quejas somáticas $\left(\mathrm{F}(2,193)=4.319, \mathrm{p}=0,015 \mathrm{\eta}^{2}=0.043\right)$, al realizar las comparaciones entre grupos utilizando el método Tukey, se obtiene que solo la diferencia entre el grupo de DT y DV es estadísticamente significativa (diff $=0,611, \mathrm{p}=0,011)$ para este síndrome. Para la escala problemas sociales $\left(\mathrm{F}(2,193)=9.598, \mathrm{p}<0,001 \mathrm{\eta}^{2}=0.090\right)$, la diferencia es estadísticamente significativa solo entre los grupos DA y DT (diff=0,974, p<0,001) . La misma tendencia se da para la escala problemas de pensamiento $(\mathrm{F}(2,193)=3.623, \mathrm{p}=0,029$ $\eta^{2}=0.036$ ), en la cual la diferencia es a favor de los alumnos con DT (diff=-0,637, $\mathrm{p}=0,033$ ). Lo mismo ocurre para la escala problemas atencionales $(\mathrm{F}(2,193)=6.792, \mathrm{p}=0.001$, $\left.\eta^{2}=0.066\right)$, con una diferencia de $0,839(\mathrm{p}=0,003)$. Por último, en el caso de conducta agresiva $\left(\mathrm{F}(2,193)=4.786, \mathrm{p}=0.009, \mathrm{\eta}^{2}=0.047\right)$ el grupo que presenta $\mathrm{DA}$ tiene diferencias significativas con los otros dos grupos, en el caso de DV, la diferencia es de 0,764 $(\mathrm{p}=0,036)$ puntos a favor de este último, mientras que la diferencia con el grupo de DT es de $0,771(p=0,007)$, a favor del grupo de DT. 


\section{Figura 2}

Escalas de Síndromes CBCL, según grupos discapacidad visual, discapacidad auditiva y desarrollo típico

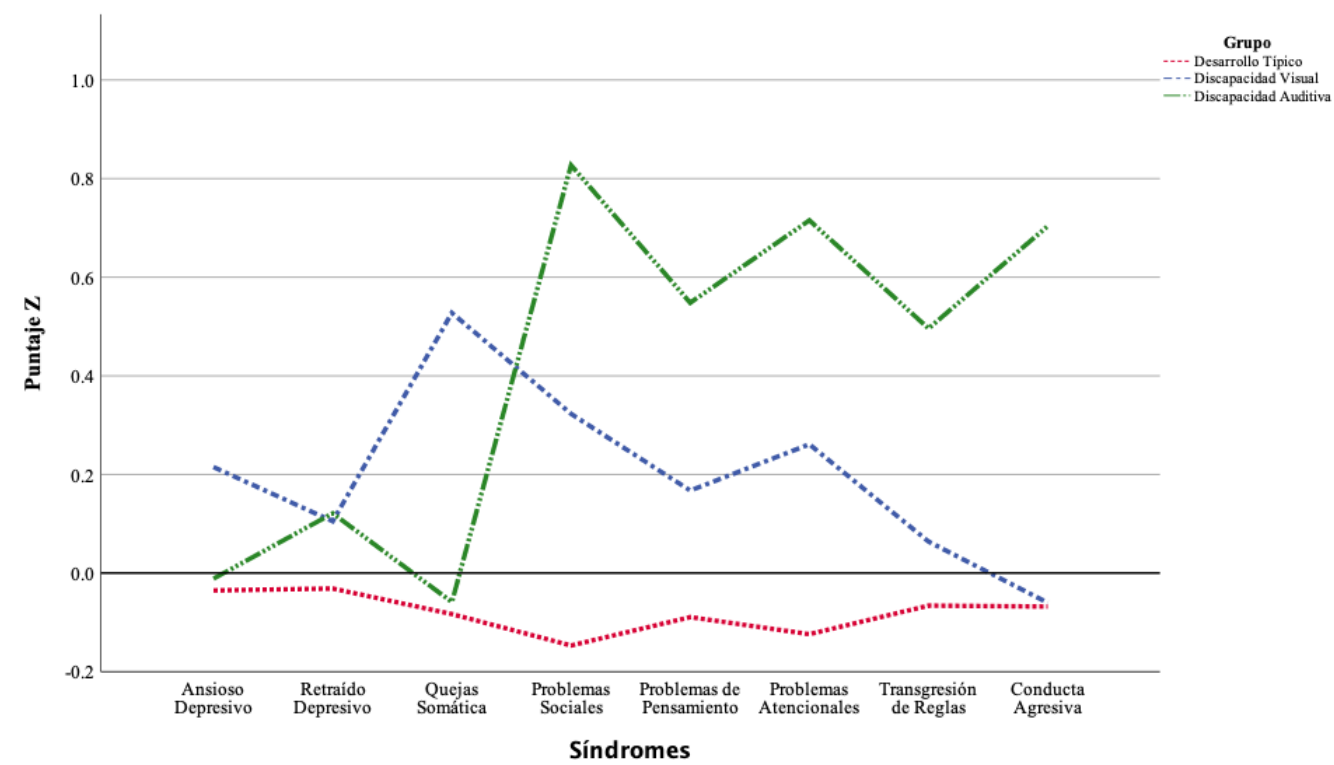

\subsection{Análisis por tipo de discapacidad}

A continuación, se presentan los resultados que buscan dilucidar si dentro de los grupos que presentan discapacidad existen diferencias de acuerdo a ciertas características que podrían influir en el estado socioemocional de los niños. Para el grupo de DA, se compara el tipo de comunicación que utilizan, es decir, oral o lengua de señas. Por su parte, el grupo que presenta DV se compara según si su grado de discapacidad es parcial o total.

Discapacidad auditiva y tipo de comunicación. Las comparaciones entre los resultados obtenidos por el grupo de alumnos con DA que utilizan el lenguaje oral o lengua de señas para comunicarse no arrojan diferencias estadísticamente significativas para las escalas generales total, externalizante e internalizante, como se observa en el Cuadro 5. Sin embargo, es relevante destacar el tamaño del efecto medio que se observa en las escalas total y de externalización.

\section{Cuadro 5}

Comparación grupo discapacidad auditiva según tipo de comunicación

\begin{tabular}{|c|c|c|c|c|c|c|c|c|c|}
\hline & \multirow{2}{*}{ GRUPO } & \multirow{2}{*}{$\mathbf{N}$} & \multirow{2}{*}{ MEDIA } & \multirow{2}{*}{ DE } & \multirow{2}{*}{ EE } & \multicolumn{2}{|c|}{ ICC } & \multirow{2}{*}{$\mathbf{T}(\mathrm{P})$} & \multirow[b]{2}{*}{ D } \\
\hline & & & & & & LI & $\mathbf{L S}$ & & \\
\hline \multirow{2}{*}{ Escala Total } & $\mathrm{CO}$ & 5 & 62,4 & 12,361 & 5,528 & \multirow{2}{*}{$-5,966$} & \multirow{2}{*}{14,599} & 0,895 & \multirow{2}{*}{0,422} \\
\hline & LSI & 12 & 58,08 & 7,513 & 2,169 & & & $(-0,385)$ & \\
\hline Escala & $\mathrm{CO}$ & 5 & 64,2 & 14,856 & 6,644 & \multirow{2}{*}{$-6,328$} & \multirow{2}{*}{17,728} & 1,01 & \multirow{2}{*}{0,470} \\
\hline Externalización & LSI & 12 & 58,5 & 8,544 & 2,466 & & & $(-0,328)$ & \\
\hline Escala & $\mathrm{CO}$ & 5 & 55,4 & 12,341 & 5,519 & \multirow{2}{*}{$-7,056$} & \multirow{2}{*}{11,19} & 0,359 & \multirow{2}{*}{0,215} \\
\hline Internalización & LSI & 12 & 53,33 & 5,726 & 1,653 & & & $(-0,735)$ & \\
\hline
\end{tabular}

Nota. $\mathrm{CO}=$ Comunicación oral, LSE = Lengua de signos, $\mathrm{N}=$ Número de participantes de la muestra total, $\mathrm{DE}=$ Desviación estándar, Error Estándar de la media, $\mathrm{ICC}=95 \%$ del Intervalo de confianza de la diferencia, LI=Límite Inferior, LS=Límite Superior. 
La Figura 3 muestra las diferencias según el tipo de comunicación utilizado por los alumnos con DA en las ocho subescalas de síndromes. Al igual que en el caso de las escalas generales, las diferencias entre los grupos no son estadísticamente significativas. No obstante, el d de Cohen para las escalas ansioso depresivo alcanza un tamaño de efecto grande para las escalas ansioso depresivo $(\mathrm{d}=0,893)$ y problemas sociales $(\mathrm{d}=0.972)$. Por su parte, las escalas problemas de pensamiento $(\mathrm{d}=0,738)$ y conducta agresiva $(\mathrm{d}=0,581)$ superan un tamaño de efecto medio.

\section{Figura 3}

Escalas de Síndromes CBCL, para grupo con discapacidad auditiva, según tipo de comunicación

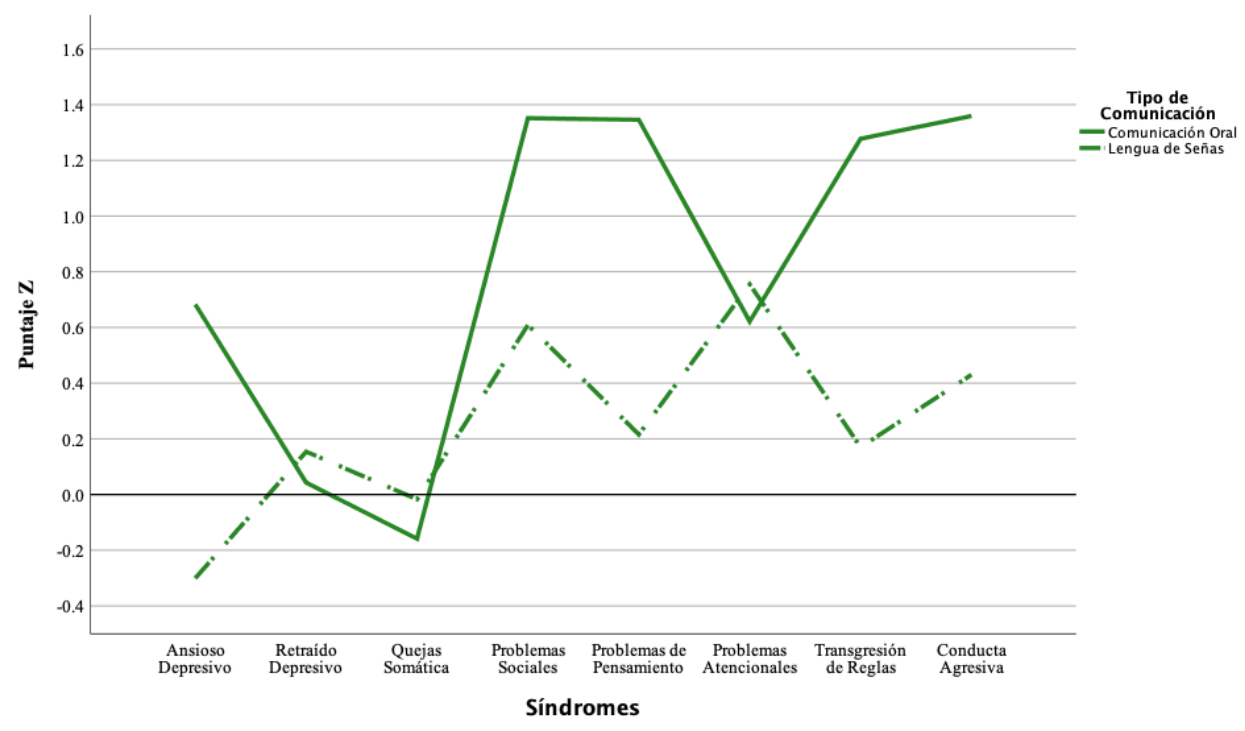

Discapacidad visual y grado de discapacidad. Respecto al grado de discapacidad que reportan los estudiantes, se obtiene que no hay diferencias significativas en ninguna de las tres subescalas mayores (internalización, externalización y total), respecto a si su discapacidad es parcial o total, como se puede apreciar en el Cuadro 6.

\section{Cuadro 6}

Comparación grupo discapacidad visual según grado de discapacidad

\begin{tabular}{|c|c|c|c|c|c|c|c|c|c|}
\hline & \multirow{2}{*}{ Grupo } & \multirow{2}{*}{$\mathbf{N}$} & \multirow{2}{*}{ Media } & \multirow{2}{*}{ DE } & \multirow{2}{*}{ EE } & \multicolumn{2}{|c|}{ ICC } & \multirow{2}{*}{$\mathrm{T}(\mathrm{P})$} & \multirow{2}{*}{ D } \\
\hline & & & & & & LI & LS & & \\
\hline \multirow{2}{*}{ Escala Total } & $\mathrm{DP}$ & 10 & 55,5 & 4,552 & 1,44 & \multirow{2}{*}{$-7,276$} & \multirow{2}{*}{4,151} & $-0,566$ & \multirow{2}{*}{0,211} \\
\hline & DTO & 16 & 57,06 & 9,427 & 2,357 & & & $(-0,577)$ & \\
\hline Escala & $\mathrm{DP}$ & 10 & 54,7 & 6,913 & 2,186 & \multirow{2}{*}{$-7,11$} & \multirow{2}{*}{5,01} & $-0,358$ & \multirow{2}{*}{0,146} \\
\hline Externalización & DTO & 16 & 55,75 & 7,497 & 1,874 & & & $(-0,724)$ & \\
\hline Escala & $\overline{\mathrm{DP}}$ & 10 & 55,9 & 10,082 & 3,188 & \multirow{2}{*}{$-8,908$} & \multirow{2}{*}{9,083} & 0,02 & \multirow{2}{*}{0,008} \\
\hline Internalización & DTO & 16 & 55,81 & 11,226 & 2,807 & & & $(-0,984)$ & \\
\hline
\end{tabular}

Nota. $\mathrm{DP}=$ Discapacidad parcial, $\mathrm{DTO}=$ Discapacidad total, $\mathrm{N}=$ Número de participantes de la muestra total, DE= Desviación estándar, Error Estándar de la media, ICC $=95 \%$ del Intervalo de confianza de la diferencia, LI=Límite Inferior, LS=Límite Superior.

La Figura 4 sintetiza los resultados obtenidos para cada uno de los síndromes de CBCL por los alumnos que presentan DV, según su grado de discapacidad. Aun cuando las diferencias entre grupos no son estadísticamente significativas para ninguna de las escalas, cabe mencionar que las escalas problemas de pensamiento $(\mathrm{d}=0,738)$ y problemas 
atencionales $(d=0,735)$ presentan un tamaño de efecto de su diferencia nominado grande, mientras las escalas ansioso depresivo $(\mathrm{d}=0,236)$, quejas somáticas $(\mathrm{d}=0,296)$ y transgresión de reglas $(\mathrm{d}=0,365)$ presentan tamaños de efecto en la diferencia entre pequeños y medianos.

\section{Figura 4}

Escalas de síndromes CBCL, para grupo con discapacidad visual, según grado de discapacidad

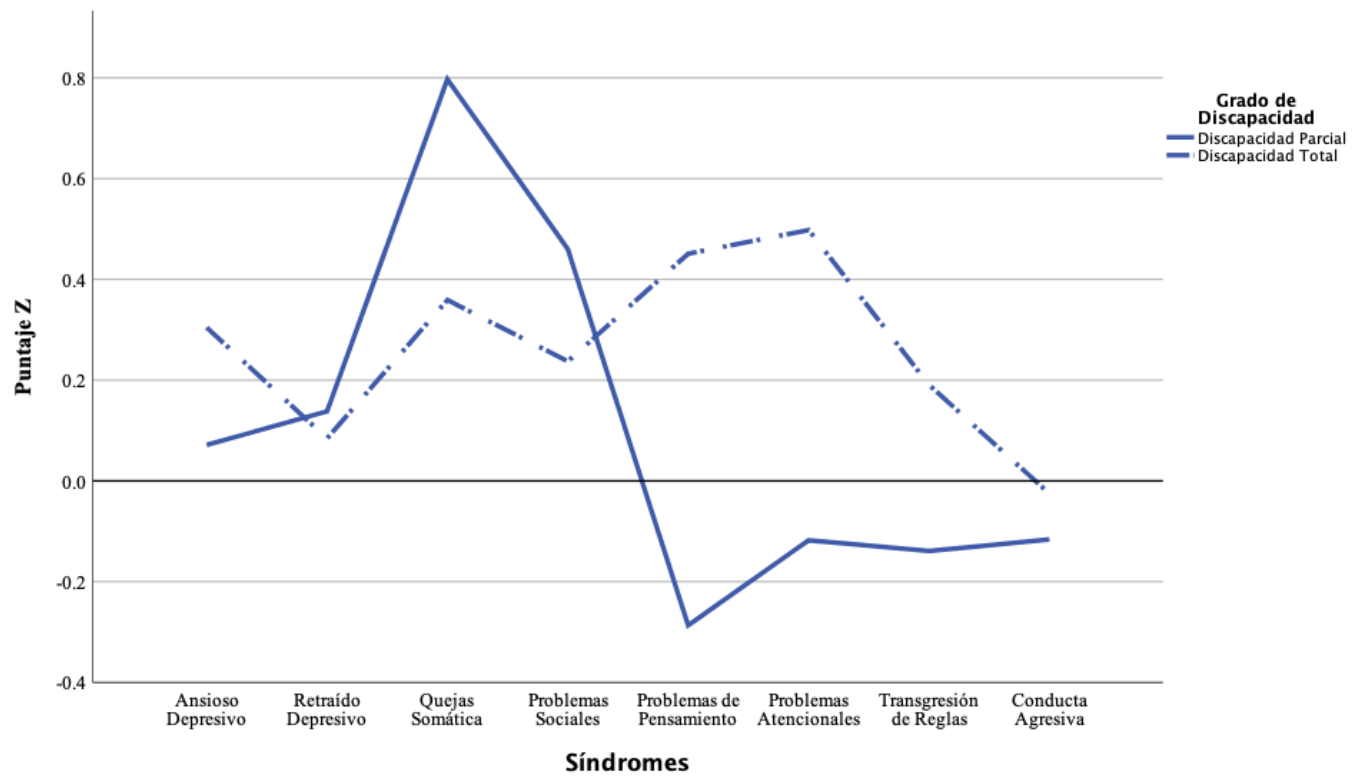

\subsection{Prevalencia de categorías diagnósticos}

A continuación, se presenta el Cuadro con la prevalencia expresada en porcentaje dentro de su grupo de pertenencia, de los criterios diagnósticos y su respectivo chi cuadrado para cada una de las escalas analizadas, según la agrupación que recoge el tipo de discapacidad. Aun cuando la frecuencia de casos no muestra diferencias estadísticamente significativas, hay ciertas tendencias que se repiten en las escalas generales. Por ejemplo, los alumnos con discapacidad parecieran tener con más frecuencia problemas de rango clínico asociados a las escalas de internalización y externalización, mientras que los alumnos con DA a la escala total.

\section{Discusión}

El objetivo general del presente estudio fue comparar los problemas emocionales y conductuales de niños con discapacidad sensorial y niños de DT. Los resultados de esta investigación indican que, tal como lo muestra la evidencia internacional los estudiantes con discapacidad sensorial manifiestan en mayor proporción que los alumnos de DT este tipo de problemas (Alimovic, 2013; Dale y Salt, 2008; Dammeyer, 2018; Maes y Grietens, 2004; Stevenson et al., 2015; Vissers y Hermans, 2018).

En respuesta a nuestra primera pregunta de investigación, los alumnos chilenos estudiados que reportan discapacidad sensorial presentan significativamente más problemas en las escalas de problemas externalizantes e internalizantes, así como en la escala total. Por otra parte, los resultados sobre escalas específicas indican que los problemas se concentran en los síndromes que aúnan quejas somáticas, problemas 
sociales, problemas de pensamiento y problemas atencionales para los alumnos que presentan discapacidad sensorial en comparación con aquellos que no presentan dicho diagnóstico.

El análisis de los resultados considerando los grupos de comparación DA, DV y DT responde a la pregunta sobre las diferencias en los problemas que presentan y su magnitud entre grupos de comparación. Se obtiene que los alumnos con DV obtienen puntajes más altos en la escala de problemas internalizantes, respecto al grupo de DT y con DA, quienes no presentan diferencias significativas entre ellos. La diferencia en problemas internalizantes entre el grupo de DT y DV también se evidencia en grupos de mayor edad, como lo hallado por Pinquart y Pfeiffer (2014), quienes encontraron diferencias en problemas emocionales entre ambos grupos. Por su parte, los alumnos con DA obtienen mayores puntajes en las escalas total y de problemas externalizantes, diferencias que son estadísticamente significativas respecto del grupo de DT y DV, quienes no presentan diferencias significativas entre ellos. La evidencia sobre mayores problemáticas de tipo total y externalizante coincide con lo hallado por van Eldik et al. (2004), salvo en lo que concierne a la escala internalizante. Por su parte, las diferencias entre el grupo de DT y que presenta DV coincide parcialmente con lo reportado por Alimovic (2013), quien encuentra diferencias además en la escala de problemas externalizantes, los cuales nosotros no hallamos.

En el caso de los niños con DA y la comparación con el resto de los grupos en síndromes específicos, se puede observar que coincidentemente con los propuesto por van Eldik et al. (2004), los niños con DA tuvieron puntajes significativamente más altos que el grupo de DT respecto de problemas sociales, problemas de pensamiento y problemas atencionales, no así del grupo que presenta DV. En el caso de la escala de conducta agresiva, los alumnos que presentan DA presentan significativamente más problemas en esta área que los otros dos grupos de comparación, lo cual se condice con el estudio de Barker y otros (2009), quienes en su ecuación estructural encuentran una relación de 0,23 entre el grado de audición y la escala de agresividad.

Respecto de los niños con DV nuestros resultados indican la presencia de diferencias significativas solo en función de las quejas somáticas, lo que coincide con lo reportado por Alimovic (2013), quien además propone diferencias relativas a los problemas sociales y a la atención, lo que no se refleja en los resultados del presente estudio.

El análisis de las escalas generales para el grupo DA considerando el tipo de comunicación que utilizan indica que no habría diferencias significativas entre ellos, sin embargo, los tamaños de efecto, de acuerdo a lo propuesto por Cohen (1992) pueden ser calificados como medios. En cuanto a las escalas específicas, al igual que para las escalas generales, las diferencias no son estadísticamente significativas, pero en esta ocasión se aprecia un tamaño de efecto grande para los síndromes ansioso-depresivo y problemas sociales y un tamaño de efecto medio para las escalas problemas de pensamiento y conducta agresiva.

La comparación entre los estudiantes con DV que presentan una ceguera total o parcial indica que no hay diferencias significativas entre los grupos para ninguna de las escalas o síndromes reportados. Sin embargo y apoyando a lo que se señala en investigaciones previas (Pinquart y Pfeiffer, 2011), hay una tendencia a que los niños y niñas con mayor grado de discapacidad, presenten mayores problemas conductuales y emocionales, lo cual se ve corroborado con tamaños de efecto medios y altos de nuestro estudio. 
En cuanto a la prevalencia de alumnos que presentarían un diagnóstico clínico, tal como lo han demostrado otros estudios, tanto los niños con DA como visual presentaron una mayor prevalencia respecto de los diagnósticos clínicos a nivel de salud mental (Dammeyer, 2009; van Eldik et al., 2004; van Gent et al., 2007). En el caso de los alumnos con DA, la presencia de rangos límites o clínicos respecto de los problemas generales es 1,8 veces más que en el caso de los niños de DT, esto coincide con los hallazgos de van Eldik y otros (2004) y van Gent y otros (2007) quienes, utilizando el mismo instrumento que en el presente estudio reportaron que los niños con DA presentaban 2,6 y 1,8 veces, respectivamente, más diagnósticos límites y clínicos en la escala de problemas totales. Sin embargo, al analizar los resultados respecto de las escalas de problemas internalizantes y externalizantes para los alumnos con DA, los resultados del presente estudio manifiestan rangos menores que el estudio de van Eldik y otros (2004), quienes reportan la presencia de un $38 \%$ de la muestra en rangos límites o clínicos, mientras que en nuestro estudio solo alcanza al 24\% (5,9\% rango límite, 17,6\% rango clínico), y rangos algo superiores que en el estudio de van Gent y otros (2007), quienes reportaron que un $19 \%$ y un $14 \%$ de los niños de la muestra presentaron resultados en rangos clínicos para las escalas de problemas internalizantes y externalizantes respectivamente. De todas formas, hay que ser cuidadosos con las comparaciones pues los estudios presentan diferencias en la edad de los participantes, mientras van Eldik y otros (2004) consideraron niños y adolescentes de entre 4 a 18 años, el estudio de van Gent y otros (2007) evaluaron adolescentes y jóvenes de entre 13 y 21 años. Nuestra muestra solo considera niños en las etapas iniciales de la escolarización.

Respecto de los niños con DV un 30,7\% de los niños evaluados presentó rangos límites o clínicos en la escala general de problemas, lo que es una proporción menor respecto de la propuesta por Tirosh y otros (1998) que describieron la presencia de problemas de comportamiento diagnosticados en un $49 \%$ de los niños de su muestra. Por otra parte, en un estudio desarrollado por Ophir-Cohen y otros. (2005) se observó que solo el $21 \%$ de los participantes presentó problemas emocionales y de comportamiento, lo que es menor al porcentaje evidenciado en el presente estudio. En ambos casos la edad de la muestra difiere de la del presente estudio, pues considera niños de entre 0 y 5 años. Las grandes diferencias observadas pueden estar relacionadas con los instrumentos de medición, que son distintos para todos los casos.

\section{Conclusiones}

El presente estudio representa un aporte a la literatura en dos sentidos. El primero es que es el único, de nuestro conocimiento, que integra el análisis de dos grupos de alumnos con discapacidad sensorial y alumnos de DT. Por otra parte, está enfocado en alumnos que están iniciando su proceso de escolarización. Ambas características permiten, iluminar el tipo de problemas que los alumnos con discapacidad sensorial enfrentan en cuanto a lo socioemocional y, además, generar intervenciones preventivas focalizadas tanto a los alumnos con discapacidad sensorial en general, como a los alumnos con DA o DV en particular.

De los resultados se puede concluir que en general los alumnos con discapacidad sensorial presentarían mayores problemas emocionales al inicio de su historia escolar, siendo los alumnos con DV quienes presentan mayores problemas de tipo internalizante mientras que los alumnos con DA presentan mayormente problemas de tipo externalizante. 
En cuanto a las limitaciones de este estudio, estas se centran en el tamaño de la muestra y en la dispersión etaria de ésta. Lo anterior afectó particularmente las comparaciones intra grupos, en el caso de alumnos con discapacidad sensorial, las cuales no arrojaron diferencias estadísticamente significativas. Estos resultados pueden ser artefacto de lo pequeña de la muestra, por lo que es necesario enfatizar la importancia de los tamaños de efecto hallados. Estos resultados dan pie para seguir explorando en las diferencias intra grupo en pos de lograr resultados más precisos y adecuados a las características de cada grupo.

Los hallazgos de este estudio arrojan información relevante que sirve de insumo para darle prioridad a la prevención y detección de problemas emocionales en grupos de riesgo. Por lo tanto, es fundamental contar con evidencia empírica que permita focalizar estrategias de tratamiento e intervención en salud mental, ya que, como se ha mencionado, los problemas emocionales se relacionan con variadas problemáticas, tanto académicas como sociales en la adolescencia y eventualmente en la adultez.

\section{Agradecimientos}

Esta investigación fue financiada por ANID PIA CIE 160007 y desarrollada por el Centro de Justicia Educacional de la Universidad Católica de Chile.

\section{Referencias}

Achenbach, T. M. y Rescorla, L. A. (2001). Manual for the ASEBA School-age forms E profiles. University of Vermont.

Alimovic, S. (2013). Emotional and behavioural problems in children with visual impairment, intellectual and multiple disabilities. Journal of Intellectual Disability Research, 57(2), 153-160. https://doi.org/10.1111/j.1365-2788.2012.01562.x

Barker, D. H., Quittner, A. L., Fink, N. E., Eisenberg, L. S., Tobey, E. A. y Niparko, J. K. (2009). Predicting behavior problems in deaf and hearing children: The influences of language, attention, and parent-child communication. Development and Psychopathology, 21(2), 373-398. https://doi.org/10.1017/So9545794090002 12.Predicting

Brauner, C. B. y Stephens, C. B. (2006). Estimating the prevalence of early childhood serious emotional/behavioral disorders: Challenges and recommendations. Public Health Reports, 121(3), 303-310. https://doi.org/10.1177/003335490612100314

Cohen, J. (1992). A power pirmer. Psychological Bulletin, 112(1)(1), 155-159.

Dale, N. y Salt, A. (2008). Social identity, autism and visual impairment (VI) in the early years. The British Journal of Visual Impairment, 26(2), 135-146. https://doi.org/10.1177/0264619607088282

Dammeyer, J. (2009). Psychosocial development in a Danish population of children with cochlear implants and deaf and hard-of-hearing children. Journal of Deaf Studies and Deaf Education, 15(1), 50-58. https://doi.org/10.1093/deafed/enp024

Dammeyer, J. (2018). Mental health and psychosocial well-being in deaf and hard-of-hearing students. En VVAA., Evidence-Based Practices in Deaf Education (pp. 437-454). Routledge. https://doi.org/10.1093/oso/9780190880545.001.0001 
Demir, T., Bolat, N., Yavuz, M., KaraçetIn, G., Doğangün, B. y Kayaalp, L. (2014a). Attachment characteristics and behavioral problems in children and adolescents with congenital blindness. Archives of Neuropsychiatry, 51(2), 116-121. https://doi.org/10.4274/npa.y6702

Demir, T., Bolat, N. yavuz, M., KaraçetIn, G., Doğangün, B. y Kayaalp, L. (2014b). Doğuştan görme engeli olan çocuk ve ergenlerde bağlanma özellikleri ve davrani\{dotless\}şsal sorunlar. Noropsikiyatri Arsivi, 51(2), 116-121. https://doi.org/10.4274/npa.y6702

Dovgan, K., Mazurek, M. O. y Hansen, J. (2019). Measurement invariance of the child behavior checklist in children with autism spectrum disorder with and without intellectual disability: Follow-up study. Research in Autism Spectrum Disorders, 58, 19-29. https://doi.org/10.1016/j.rasd.2018.11.009

Dyck, M. J., Farrugia, C., Shochet, I. M. y Holmes-Brown, M. (2004). Emotion recognition/understanding ability in hearing or vision-impaired children: Do sounds, sights, or words make the difference? Journal of Child Psychology and Psychiatry and Allied Disciplines, 45(4), 789-800. https://doi.org/10.1111/j.1469-7610.2004.00272.x

Eisenberg, N. (2006). Introduction. En W. Damon, R. Lerner y N. Eisenberg (Eds.), Handbook of child psychology: Social, emotional and personality development (pp. 1-23).Wiley.

Fernández-Berrocal, P. y Ruiz Aranda, D. (2008). La inteligencia emocional en la educación. Electronic Journal of Research in Educational Psychology, 6(15), 42 1-436.

Goldenberg, I., Matheson, K. y Mantler, J. (2006). The assessment of emotional intelligence: A comparision of permormance-based and self-report methodologies. Journal of Personality Assessment, 86(1), 33-45. https://doi.org/10.1207/s 15327752jpa860 1

Hintermair, M. (2014). Psychosocial development in deaf and hard of hearing children in the twenty first century. In M. Marschark, G. Tang y H. Knoors (Eds.), Bilingualism and bilingual deafeducation (pp. 152-186). University Press.

Huber, M. y Kipman, U. (2011). The mental health of deaf adolescents with cochlear implants compared to their hearing peers. International Journal of Audiology, 5o(3), 146-154. https://doi.org/10.3109/14992027.2010.533704

Huurre, T. M. y Aro, H. M. (1998). Psychosocial development among adolescents with visual impairment. European Child and Adolescent Psychiatry, 7(2), 73-78. https://doi.org/10.1007/s007870050050

Ivanova, M. Y., Achenbach, T. M., Dumenci, L., Rescorla, L. A., Almqvist, F., Weintraub, S., ... Verhulst, F. C. (2007). Testing the 8-syndrome structure of the child behavior checklist in 30 societies. Journal of Clinical Child and Adolescent Psychology, 36(3), 405-41 7.

https://doi.org/10.1080/15374410701444363

Jones, P. B. (2013). Adult mental health disorders and their age at onset. British Journal of Psychiatry, 202(54), 5-11. https://doi.org/10.1192/bjp.bp.112.119164

Khan, S., Edwards, L. y Langdon, D. (2005). The cognition and behaviour of children with cochlear implants, children with hearing aids and their hearing peers: A comparison. Audiology and Neurotology, 1O(2), 117-126. https://doi.org/10.1159/000083367

Lecannelier, F., Ewert, J. C. P., Groissman, S., Gallardo, D., Bardet, A. M., Bascuñan, A. y Rodríguez, J. (2014). Validación del inventario de conductas infantiles para niños de entre 11/2-5 años (CBCL 11/2-5) en la Ciudad de Santiago de Chile. Universitas Psychologica, 13(2), 491-500. https://doi.org/10.11144/Javeriana.UPSY13-2.vici

Leiva Bahamondes, L. y Andrade, R. R. (2018). Validación de la escala de problemas emocionales y del comportamiento youth self report (YSR/11-18) en una muestra de adolescentes chilenos. Terapia Psicológica, 36, 23-32. 
Lopes, P. N., Salovey, P., Côté, S. y Beers, M. (2005). Emotion regulation abilities and the quality of social interaction. https://doi.org/10.1037/1528-3542.5.1.113

Maes, B. y Grietens, H. (2004). Parent-reported problem behavior among children with sensory disabilities attending elementary regular schools. Journal of Developmental and Physical Disabilities, 16(4), 361-375. https://doi.org/10.1007/s10882-004-0692-9

Mesman, J., Bongers, I. L. y Koot, H. M. (2001). Preschool developmental pathways to preadolescent internalizing and externalizing problems. Journal of Child Psychology and Psychiatry and Allied Disciplines, 42(5), 679-689. https://doi.org/10.1017/So021963001007351

Ophir-Cohen, M., Ashkenazy, E., Cohen, A. y Tirosh, E. (2005). Emotional status and development in children who are visually impaired. Journal of Visual Impairment and Blindness, 99(8), 478485. https://doi.org/10.1177/0145482x0509900804

Pinquart, M. y Pfeiffer, J. P. (2011). Psychological well-being in visually impaired and unimpaired individuals: A meta-analysis. The British Journal of Visual Impairment, 29(1), 27-45. https://doi.org/10.1177/0264619610389572

Pinquart, M. y Pfeiffer, J. P. (2014). Change in psychological problems of adolescents with and without visual impairment. European Child and Adolescent Psychiatry, 23(7), 571-578. https://doi.org/10.1007/s00787-013-0482-y

Reynolds, S. A., Fernald, L. C. H., Deardorff, J. y Behrman, J. R. (2018). Family structure and child development in Chile: A longitudinal analysis of household transitions involving fathers and grandparents. Demographic Research, 38(1), 1777-1814. https://doi.org/10.4054/DemRes.2018.38.58

Roch-Levecq, A. C. (2006). Production of basic emotions by children with congenital blindness: Evidence for the embodiment of theory of mind. British Journal of Developmental Psychology, 24(3), 507-528. https://doi.org/10.1348/026151005X50663

Rocha, M. M., Rescorla, L. A., Emerich, D. R., Silvares, E. F. M., Borsa, J. C., Araújo, L. G. S., Assis, S. G. (2013). Behavioural/emotional problems in Brazilian children: Findings from parents' reports on the child behavior checklist. Epidemiology and Psychiatric Sciences, 22(4), 329-338. https://doi.org/10.1017/S2045796012000637

Runjić, T., Prcić, A. B. y Alimović, S. (2015). The relationship between social skills and behavioral problems in children with visual impairment. Runjic, Tina Bilic Pricic, Ante Alimovic, Sonja, 51(2), 64-76.

Santa-Cruz, C. y Hohlberg, E. (2019). La adaptación de una herramienta tecnológica para evaluar funciones ejecutivas en niños con discapacidad visual ${ }_{\mathrm{C} E l}$ cambio de modalidad afecta su capacidad de discriminación?. Pensamiento Educativo. Revista de Investigación Educacional Latinoamericana, 56(2), 1-18.

Stevenson, J., Kreppner, J., Pimperton, H., Worsfold, S. y Kennedy, C. (2015). Emotional and behavioural difficulties in children and adolescents with hearing impairment: A systematic review and meta-analysis. European Child and Adolescent Psychiatry, 24(5), 477-496. https://doi.org/10.1007/s00787-015-0697-1

Tirosh, E., Shnitzer, M. R., Davidovitch, M. y Cohen, A. (1998). Behavioural problems among visually impaired between 6 months and 5 years. International Journal of Rehabilitation Research, 21, 63-70.

Tröster, H. y Brambring, M. (1992). Child: Care health and development. Child: Care, Health and Development, 18, 207-227. https://doi.org/10.1111/j.1365-2214.2004.00488.x 
Naciones Unidas. (2014). Mental health matters. Social inclusion of youth with mental conditions. Departmen of Economic and Social Affairs.

van Eldik, T., Treffers, P. D. A., Veerman, J. W. y Verhulst, F. C. (2004). Mental health problems of deaf dutch children. American Annals of the Deaf, 148(5), 390-395.

van Gent, T., Goedhart, A. W., Hindley, P. A. y Treffers, P. D. A. (2007). Prevalence and correlates of psychopathology in a sample of deaf adolescents. Journal of Child Psychology and Psychiatry and Allied Disciplines, 48(9), 950-958. https://doi.org/10.1111/j.1469-7610.2007.01775.x

Vicente, B., De La Barra, F., Saldivia, S., Kohn, R., Rioseco, P. y Melipillan, R. (2012). Prevalence of child and adolescent psychiatric disorders in Santiago, Chile: A community epidemiological study. Social Psychiatry and Psychiatric Epidemiology, 47(7), 1099-1109. https://doi.org/10.1007/s00127-011-0415-3

Vissers, C. T. W. M. y Hermans, D. (2018). Social-emotional problems in deaf and hard-of-hearing children from an executive and theory-of-mind perspective. En VVAA., Evidence-based practices in deaf education (pp. 437-454). Routledge.

https://doi.org/10.1093/oso/9780190880545.001.0001

Wright, D. y Torrey, G. K. (2000). A comparison of two peer-referenced assessment techniques with parent and teacher ratings of social skills and problem behaviors. Behavioral Disorders, 26(2), 173-182. https://doi.org/10.1177/019874290102600207

\section{Breve CV de las autoras}

\section{Catalina Santa Cruz}

Psicóloga de la Pontificia Universidad Católica de Chile. Doctora en Psicología de la Pontificia Universidad Católica de Chile. Investigadora asociada del Centro de Justicia Educacional UC. en la línea de investigación denominada "Inclusión de la discapacidad: Considerando la Diversidad en Poblaciones Diversas: De las Políticas a las Intervenciones en Educación Especial”. Sus áreas de interés son trayectorias de aprendizaje, alumnos con discapacidad, educación preescolar, desarrollo cognitivo y socioemocional. Ha realizado estudios sobre trayectorias de aprendizaje de alumnos con discapacidad sensorial y además, adaptaciones de instrumentos de evaluación cognitiva para alumnos con necesidades educativas especiales. ORCID ID: https://orcid.org/0000-0002-1074-6207. Email: csantacr@uc.cl

\section{Victoria Espinoza}

Directora de Investigación del Centro de Desarrollo de Tecnologías de Inclusión y colaboradora en la línea de Investigación y Discapacidad del Centro de Justicia Educacional. Profesora de Educación Básica especialista en Dificultades de Aprendizaje e Inclusión Educativa, Magíster en Psicología, mención en Psicología Educacional, Doctora en Psicología de la Pontificia Universidad Católica de Chile. Sus temas de investigación refieren al proceso de adquisición de la lectura, la educación inicial, el desarrollo de las funciones ejecutivas, la inclusión educativa de estudiantes con discapacidad y las brechas de aprendizaje terminadas por el nivel socioeconómico y su impacto en las posteriores trayectorias de aprendizaje. ORCID ID: https://orcid.org/0000-0001-9462-1 139. Email: victoriaespinoza@uc.cl 


\section{Elisa Hohlberg}

Psicóloga Orchard College. Titulada en Psicología de la Pontificia Universidad Católica de Chile. Sus áreas de interés son la educación inclusiva, competencias socioemocionales, generación y estandarización de instrumentos de evaluación inclusivos, trayectorias del desarrollo cognitivo, socioemocional y lenguaje en estudiantes con y sin discapacidad, neuropsicología, instrumentos de evaluación inclusivos y aplicación del modelo de Rash para la estandarización de instrumentos de evaluación desde una perspectiva del desarrollo. Actualmente se desempeña como psicóloga en el Colegio Orchard y previamente como coordinadora de terreno de la Línea de Inclusión de la Discapacidad del Centro de Justicia Educacional UC. ORCID ID: https://orcid.org/O000-0003-22156789. Email: mehohlberg@uc.cl 